\title{
Adopting a robust attitude: risk-constrained decisions and knowledge acquisition
}

\author{
William Fauriat \\ Université Paris-Saclay, CentraleSupélec, Laboratoire Genie Industriel, 3 rue Joliot-Curie 91190 \\ Gif-sur-Yvette,France E-mail: william.fauriat@centralesupelec.fr
}

Good decisions, in design or operation stages, positively impact system performances and can prevent it form suffering undesired scenarios and their consequences, in a uncertain environment. Using Robust Optimization (RO), a so-called chance-constraint, here denoted as risk-constraint as it embeds the preferences of the decision maker with respect to risk tolerance, can be enforced to ensure that the obtained solution of the decision problem is acceptable in this regard. Yet, this solution will only be as good as the knowledge of the uncertain environment that is used in the decision-making process. In this paper, we study the possibility for the decision-maker to refine its knowledge of the system in its operating environment, through information collection. The concept of Value of Information (VoI) is used to quantify potential improvements in decisions outcomes that may be achieved through a modification of the state of knowledge and a reduction in uncertainty. In particular, using decisions that are conditioned on an environment with reduced uncertainty, it is often possible to lower the level of risk for a given total operating cost (or Value-at-Risk) or vice versa. Hence, in a wide sense, knowledge acquisition may represent a step towards adopting a more robust attitude, with respect to risk, when making decisions.

Keywords: Value of Information, Robust Optimization, decision-making under uncertainty, chance-constrained optimization, model uncertainty, Value-at-Risk.

\section{Introduction}

Decision-making under uncertainty, whether in design or operation stages and for a complex product or system, is an inherently risky endeavor. It may prove hard to build a stochastic description that is representative of such uncertainty, see e.g. Cox Jr (2012). This is especially true for systems which behavior is becoming ever more difficult to model because of the increase in structural complexity or in the diversity of interactions between its constituting elements and with the external environment. Yet, decisions that determine system performances and either prevent it from suffering undesired scenarios and their consequences, or fail to do so, are only as good and relevant in practice, as the knowledge of the uncertain environment that is built into the decision-making process from which there are computed.

In the framework of Robust Optimization (RO) Mulvey et al. (1995), a chance-constraint Charnes and Cooper (1959) can be enforced during the search for a solution of the decision problem. This mathematical scheme is used to ensure that despite "known variations" of noncontrollable (i.e. uncertain) parameters, the obtained solution guarantees that the system operates within acceptable boundaries. The preferences of the decision-maker regarding its degree of tolerance for the probabilities of experiencing the consequences of undesired scenarios, i.e. its attitude towards risk, see e.g. Kaplan and Garrick
(1981); Bedford et al. (2001); Aven (2012), can be embedded in this chance-constraint. Hence, it is described here as a "risk-constraint" and associated to a robust solution of the decision problem.

However this solution is, by construction, dependent on the knowledge of the decision-maker with respect to the uncertain parameters of the problem. In particular, if the decision-maker wishes to shield himself from undesired outcomes on which there is little information or against a wide range of uncertain scenarios, the solution might prove quite costly.

In this paper, we study the possibility for the decision-maker to refine its knowledge and understanding of the behavior of the system in its operating environment, through information collection, otherwise conceivable as knowledge acquisition, before coming to a decision.

The concept of Value of Information (VoI) Raiffa (1961); Howard (1966), built on core principles from both decision theory and Bayesian theory, is used here to quantify potential improvements in decisions outcomes that may be achieved through a modification of the state of knowledge and a reduction in uncertainty, see also recent applications in e.g. Pozzi and Der Kiureghian (2011); Straub (2014); Zonta et al. (2014); Memarzadeh and Pozzi (2016); Fauriat and Zio (2018). In the context of this paper, it is proposed to compute VoI by comparing chance-constrained optimization results from both unconditional and conditional (on the collected information) descrip-

Proceedings of the 30th European Safety and Reliability Conference and the 15th Probabilistic Safety Assessment and Management Conference. Edited by Piero Baraldi, Francesco Di Maio and Enrico Zio Copyright (C) 2020 by ESREL 2020 PSAM 15 Organizers. Published by Research Publishing, Singapore ISBN: 981-973-0000-00-0 :: doi: 10.3850/981-973-0000-00-0_Fauriat'ESREL2020 v0 
tions of the uncertain environment of the decision problem.

The paper is organized as follows. In section 2, the concept of RO is discussed, with the objective of making risk-constrained decisions in an uncertain environment. The notion of VoI is then introduced in section 3 and its use for evaluating the benefits of uncertainty reduction, or knowledge acquisition, is considered in the context of RO problems. A simple example made of a network with a supply and demand planning problem is presented in section 4 to illustrate the proposed methodological framework employing VoI for robust decision-making. Using the previous example, the interest of this methodological framework is discussed with more details in section 5, along with the difficulties that have to be addressed to deploy it. Conclusions are summarized in section 6.

\section{Robust or 'risk-constrained' optimization}

\subsection{Problem solving and decision-making}

For most engineering or management applications, dealing with uncertainty, non-controllable factors and risk, whether for the design or the operation of a given product or system, is a crucial part of the issue of determining an appropriate course of action. Multiple perspectives can be adopted in that regard, from operation research Mulvey et al. (1995), to risk analysis and management Bedford et al. (2001); Aven (2012), to statistical decision theory Raiffa (1961); Savage (1972); Berger (2013). Under that last framework, determining such a course of action, otherwise known as making a decision, can be cast mathematically as the resolution of a loss minimization problem (or equivalently a utility maximization problem), i.e. an optimization problem, generally with both decision parameters $a \in \mathcal{A}$ (action space) and random parameters $\theta \in \Theta$ (parameter space).

\subsection{Risk and decision-making}

As the definition of risk and robustness may not be universally accepted, let us start by precising the position adopted here.

In Kaplan and Garrick (1981), risk is defined as the set of triplets $R=\left\{s_{j}, p_{j}, x_{j}\right\}$ composed of $j=1, \ldots, N$ scenarios $s_{j}$ of probability $p_{j}$ and associated to a consequence quantified by a numerical damage $x_{j}$. In Savage (1972), in decision matrices, or so-called "small world representations", the decision-maker may choose between different course of actions $a_{i}$ which will produce different consequences, let us simplify by associating them a numerical value through a loss function
$L\left(\theta_{j}, a_{i}\right)$, for different possible realizations of the state of the world $\theta_{j}$. In this latter framework, one may also think of risk description as the specification of a set of associations between consequences and their related probability of occurrence. Let us note that this set of associations, or in mathematical terms, this lottery $\left\{p_{j}, L\left(\theta_{j}, a_{i}\right)\right\}_{j=1, \ldots, N}$, is fully determined once a course of action $a_{i}$ has been fixed.

From that point on, there is no universal solution regarding which course of action (which lottery) is preferable. Using the example of finance terminology, some decision-makers, generally described as risk-adverse, may prefer decisions (lotteries) that tradeoff a lower average payoff for a lower possibility of experiencing scenarios with heavy losses, or vice versa for riskseekers. In simple terms, in the presence of uncertainty, adopting a position towards risk means selecting an acceptable lottery, among all possible choices (possibly uncountable), in regards of one's own preferences.

Two popular decisions rule are considered in what follows. The Maximum Expected Utility (MEU) rule consists in selecting the action corresponding to the highest expected payoff:

$$
a^{*}=\underset{a \in \mathcal{A}}{\arg \max }\left(-E_{\theta}[L(\theta, a)]\right)
$$

where $U=-L$, as loss can be seen as negative utility and $E_{\theta}[$.$] is the mathematical expectation$ over all possible states of the world. The minimax, or worst-case rule (for a finite number of scenarios) may yield a solution with a lower expected payoff but which is generally better protected against heavy losses:

$$
a^{*}=\underset{a \in \mathcal{A}}{\arg \min }\left(\max _{\theta \in \Theta} L(\theta, a)\right)
$$

\subsection{Risk-constrained or robust decisions}

According to the perception of the notion of robustness that is discussed in Mulvey et al. (1995), an intermediate rule between both alternatives above is, for example, to control the probability of occurrence, or risk level $\alpha$, of scenarios that are deemed unacceptable. To the this end, a chance-constraint is enforced and the problem is framed as a chance-constrained optimization Charnes and Cooper (1959):

$$
\left\{\begin{array}{l}
\min _{a \in \mathcal{A}}\left(E_{\theta}[L(\theta, a)]\right) \\
\text { subject to } \\
\operatorname{Pr}[g(\theta, a)<0]<\alpha
\end{array}\right.
$$

where $g(\theta, a)<0$ is a constraint that "should not be violated" with a probability superior to $\alpha$. Thus, a "robust" solution may be conceived as a 
compromise between a search for a sufficiently low average cost and a limitation of the occurrence (or related probability), given the non-controllable parameters of the problem, of constraint violations that are often synonymous with penalties. One may also speak of solutions that are "feasible most of the time".

Without dwelling too much into the mathematical details of RO, as it is not the core purpose of this paper, a possible illustration of different decision rules is given on Figure 1. In particular, for the example of a RO problem based on a single decision parameter $a \in \mathbb{R}$ and expressing the socalled $\alpha$ - quantile of the loss distribution, or Value-at-Risk $\alpha, q^{\alpha}(a)=\operatorname{VaR}_{\alpha}(a)$ defined by:

$$
\operatorname{Pr}\left[L(\theta, a)>\operatorname{VaR}_{\alpha}(a)\right]=\alpha
$$

results corresponding to $\alpha=1 \%$ and $\alpha=5 \%$ are illustrated on Figure 1 (green dotted lines).

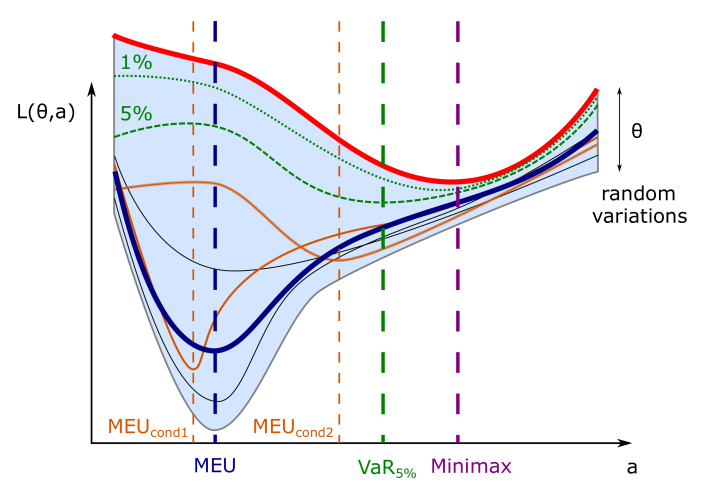

Fig. 1. Illustration of a RO problem. Solutions associated to different decision rules (different lotteries) are given (vertical lines), namely for MEU, Minimax, $\operatorname{VaR}_{\alpha}$. The thick blue line gives the expected loss. The thick red line represents a worst-case scenario. The shaded area is where most of the probability distribution of loss resides (for different values of $a$, i.e. different lotteries).

If a closed-form expression can be obtained for $\operatorname{VaR}_{\alpha}(a)$, as is the case for the simple example presented in section 4 a decision rule may be based on minimizing such quantity, thus giving some control about the maximal amount one agrees to pay, with probability $1-\alpha$. In this paper, the minimization of $\mathrm{VaR}_{\alpha}$ is considered as a riskconstrained optimization process and this decision rule is selected for the discussion on knowledge improvement in what follows.

\section{Uncertainty reduction and Value of Information}

\subsection{Value of Information (VoI)}

The concept of Value of Information (VoI) Raiffa (1961); Howard (1966) is built on core principles from both decision theory and Bayesian theory. Simply put, the idea is to provide a rational approach to attach a value to a piece of information, depending on its ability to "guide our decision" Pozzi and Der Kiureghian (2011) to achieve "better outcomes". VoI can be used to evaluate the benefit of reducing uncertainty in a decisionmaking context.

Formally, VoI is computed as the difference between the outcomes, as valued by a utility measure, that are achieved with and without additional information. If actions are selected using a MEU rule, VoI may be computed using:

$\mathrm{VoI}=\min _{a} E_{\theta}[L(\theta, a)]-E_{z}\left[\min _{a} E_{\theta \mid z}[L(\theta, a)]\right]$

where $E_{\theta \mid z}[$.$] is the mathematical expectation on$ the conditional distribution $\theta \mid z$ and $z$ is a collected piece of information, which, once known, "restrains" the possible variation of $\theta$ and thus reduces uncertainty. With explicit formulation of all involved distribution functions, it reads:

$$
\begin{aligned}
& \mathrm{VoI}=\min _{a \in A} \int L(\theta, a) f(\theta) d \theta \\
& -\int\left(\min _{a \in A} \int L(\theta, a) f(\theta \mid z) d \theta\right) f(z) d z
\end{aligned}
$$

The first term of equation (5) is the average outcome of the optimal unconditional decision, or said differently, the average outcome associated to the best decision in the MEU sense, given the prior knowledge on the state of the world, expressed through $f(\theta)$. The second term corresponds to the average, over $f(z)$, of all optimal conditional decisions given one collected observation $z$. Often, a more "tailored" course of action can be selected, provided it is available in the action space $\mathcal{A}$, for an environment with reduced uncertainty, expressed through $f(\theta \mid z)$. If so, there is a potential for gain, on average, in taking decisions conditionally rather than based solely on the prior knowledge. The VoI metric is an evaluation of that potential for gain and improvement in decision outcomes that is achievable through a reduction of uncertainty. More details can be found in e.g. Raiffa (1961).

On Figure 1 the two yellow lines illustrate conditional expected losses for two possible realizations $z_{1}$ and $z_{2}$ of the collected observation. The two vertical dotted yellow lines determine two 
conditional decisions $\mathrm{MEU}_{\text {cond } 1}$ and $\mathrm{MEU}_{\text {cond } 2}$ that, taken on average, i.e. for multiple possible values of $z \sim f(z)$, may correspond to a lower expected loss.

\subsection{Use of VoI for robust decision-making}

Often, VoI is used as a decision-support metric and compared against the cost of collecting information, for example, through a structural health monitoring (SHM) device or procedure, see e.g. Pozzi and Der Kiureghian (2011); Zonta et al. (2014), or through planned inspections, see e.g. Fauriat and Zio (2018). Naturally, information is only "worth collecting" if its collection cost does not exceed its benefits.

In this paper, we propose to see VoI as a means to question the robustness of our decision-making process and inquire about the relevance of refining our knowledge before making a decision. In particular, we compare the robust solutions, for any given risk level $\alpha$, that are both obtained with and without the collection of additional information, i.e. with or without a reduction of uncertainty. If $q_{\theta}^{\alpha}[]=.\operatorname{VaR}_{\alpha}[$.$] is used to describe the \alpha-$ quantile of the loss distribution, where the subscript $\theta$ recalls the dependency on the uncertain environment, one may compute VoI with:

$\mathrm{VoI}=\min _{a} q_{\theta}^{\alpha}[L(\theta, a)]-E_{z}\left[\min _{a} q_{\theta \mid z}^{\alpha}[L(\theta, a)]\right]$

If robustness is perceived as linked to the ability of the decision-maker to control the risk level associated to different possible courses of action, undertaken in an uncertain environment, i.e. in the presence of non-controllable parameters, then making sure that the decision is based on the appropriate body of knowledge is synonymous with an increase in robustness, that is, an increase on the control of risk. As a concrete illustration, note that if optimal conditional outcomes correspond to lower cost values than the optimal unconditional outcome, it may be possible to trade such potential for gain for a tightening of risk control (i.e. a gain robustness in practice).

\section{Application example}

\subsection{Supply and demand network planning}

To illustrate the application of VoI to a simple RO problem, let us consider the following example. A simple transportation network is composed of three lines connecting two end destinations. On each line, there is a level of demand for transportation that cannot be known exactly at planning time. The operator of the network can affect resources in the form of transportation units, e.g. trains, on each one of the different lines.

For this simple problem, the decision parameters are the number of transportation units $a=\left(a_{1}, a_{2}, a_{3}\right)$ to supply on each line, while the uncertain parameters are the level of the demand on each line $\theta=\left(d_{1}, d_{2}, d_{3}\right)$. Each demand value follows a Gaussian distribution with known parameters $d_{i} \sim N\left(\mu_{i}, \sigma_{i}\right)$ for $i=1, \ldots, 3$.

In this simple case, a closed-form expression can be obtained for the $\alpha$ - quantile or Value-atRisk $q_{\theta}^{\alpha}=\mathrm{VaR}_{\alpha}$ :

$$
\begin{aligned}
& q_{\theta}^{\alpha}(a)=c_{t}\left(a_{1}+a_{2}+a_{3}\right)+c_{b} \frac{1-\lambda^{a_{1}+a_{2}+a_{3}}}{1-\lambda} \\
& +\nu \min \left[-\Phi^{-1}\left(1-\alpha^{1 / 3}, \frac{a_{1}-\mu_{1}}{\sigma_{1}}\right), 0\right] \\
& \quad+\nu \min \left[-\Phi^{-1}\left(1-\alpha^{1 / 3}, \frac{a_{2}-\mu_{2}}{\sigma_{2}}\right), 0\right] \\
& +\nu \min \left[-\Phi^{-1}\left(1-\alpha^{1 / 3}, \frac{a_{3}-\mu_{3}}{\sigma_{3}}\right), 0\right]
\end{aligned}
$$

where $c_{t}$ is the cost of the affectation of one transportation unit on any line, $c_{b}$ is the cost of buying one transportation unit and $\lambda$ is the exponential increase in investment cost when buying units, $\nu$ is a proportional penalty term for each extra unsatisfied units of demand. $\Phi^{-1}(u, v)$ is the inverse cumulative density function of the Gaussian distribution at quartile $u$ and with $v$ as a normalized variable and $\Phi^{-1}\left(1-\alpha^{1 / 3}, v\right)$ gives the number of unsatisfied units in excess of quartile $\left(1-\alpha^{1 / 3}\right)$ for one of the three demand distributions, taken independently, that if summed for the three distributions then "cover" quartile $\alpha$ for the total cost on all lines.

Details of the illustration example are not crucial as many different applications cases could be considered. Let us instead insist on the fact that here, the problem is made computationally friendly thanks to the closed-form expression of the Value-at-Risk $\alpha$ in (8).

On Figure 2 we display an illustration of the realization of random cost values $L\left(\theta_{j}, a\right)$ and of VaRs corresponding to different $\alpha$, for a proportional penalty and for the cost parameters described above. When varying $a_{2}$, fixed values are taken for $a_{1}$ and $a_{3}$, namely $a_{1}^{0}$ and $a_{3}^{0}$.

\subsection{Application and computation of VoI}

The computation of VoI, as defined in (7) can be performed based on (8) and through the use of a simple optimization scheme, as $q_{\theta}^{\alpha}(a)$ and $q_{\theta \mid z}^{\alpha}(a)$ happen to be convex, see Figure 2 .

In order to study the effect of knowledge acquisition and uncertainty reduction on the outcome of RO-based decisions, multiple conditional opti- 


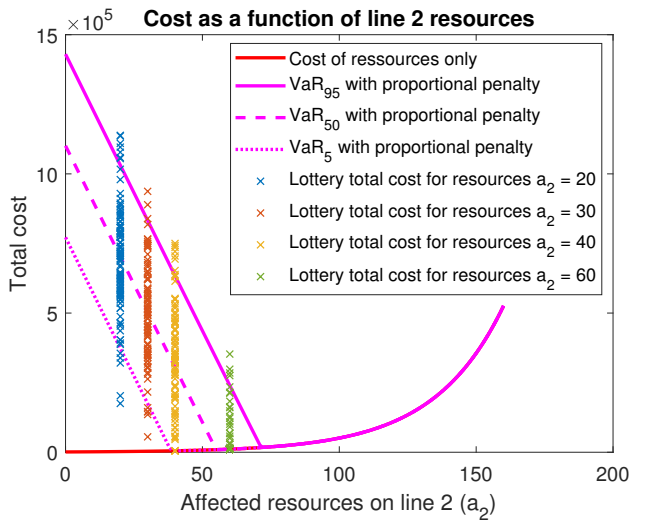

Fig. 2. Illustration of resources and penalty costs along the $a_{2}$ axis. This figure is the equivalent of Figure 1 for the example considered here.

mizations (linked to collected observations $z$ ) are carried out.

For each line $i=1, \ldots, 3$, assuming that $\mathrm{VoI}$ is calculated in relation with information collected on one line at a time, $\mu_{z, i}=z_{i}$ is sampled directly from $z_{i}=N\left(\mu_{i}, \sigma_{i}\right)$ and $\sigma_{z, i}=\kappa \sigma_{i}$, where $\kappa$ determines the uncertainty reduction factor. Those conditional parameters $\left(\mu_{z, i}, \sigma_{z, i}\right)$ are then substituted in the VaR expression in (8), thus yielding $q_{\theta \mid z}^{\alpha}(a)$. Also, we may write:

$$
d_{i} \mid z_{i} \sim N\left(z_{i}, \kappa \sigma_{i}\right)
$$

The lower $\kappa$ is, the less uncertainty we have on the true value of the demand $d_{i}$, prior to making a decision. The value $\kappa$ is an embodiment of the quality of our information collection or knowledge acquisition process.

Sampling-based numerical integration with $N=1000$ repetitions is carried out to simulate information collection, conditional RO and eventually estimation of VoI. Results for the different conditional optimizations are given on Figure 3. As a general illustration, here for this simple problem, it is possible to save from 30 to 55 percent on operation and investment costs, if uncertainty on $d_{2}$ is reduced by $90 \%$, while conserving the same level of risk control $\alpha$. Simply put, one may remain just as conservative but with a lower operation cost by taking advantage of knowledge acquisition (which cost will also have to be taken into account).

\section{Further analysis and discussion}

\subsection{Variaton of VoI with risk coverage and uncertainty reduction}

The computation of $\mathrm{VoI}$ is repeated for varying level of risk (with fixed $\kappa=0.1$ ), i.e. associated
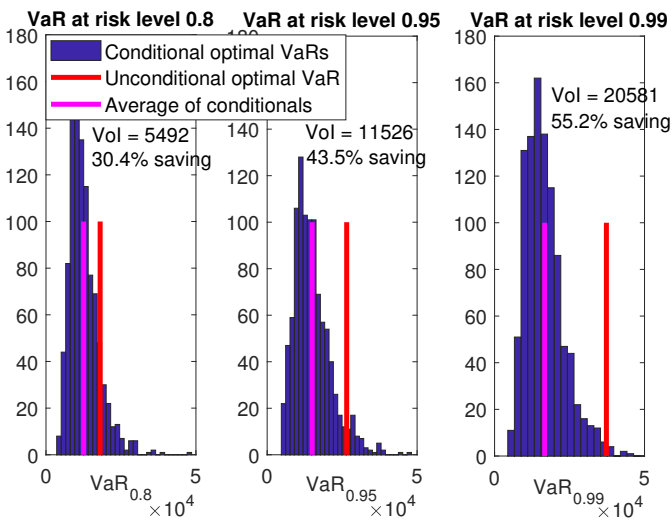

Fig. 3. Computation of unconditional and conditional optimal $\mathrm{VaR}_{\alpha}$ for different risk levels $\alpha$ and computation of VoI. Results are given here for information collected on line 2, i.e. on $d_{2}$, with a reduction of uncertainty of $90 \%$, i.e. $\kappa=0.1$

to more or less conservatives or risk-adverse attitudes, and displayed on Figure 4
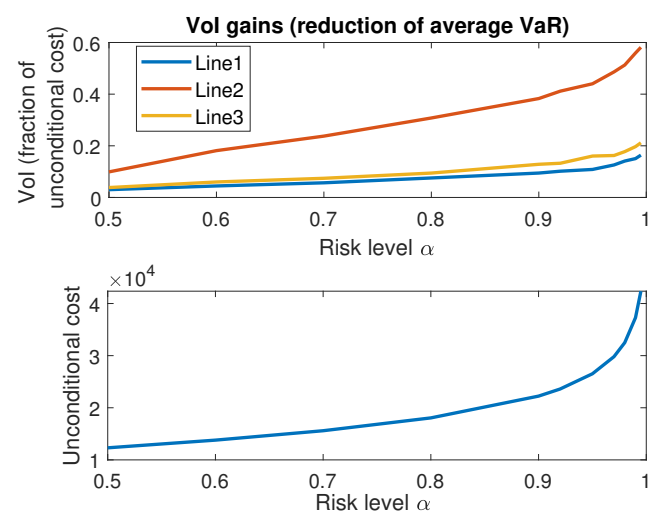

Fig. 4. Evolution of VaR with varying risk level for an unconditional decision (bottom figure). Evolution of potential gains, as measured by relative $\mathrm{VoI}$, for a varying level of risk coverage (top figure). For example, a 0.5 value on the top figure corresponds to a $50 \%$ reduction of the cost (VaR), with respect to the unconditional reference.

It is seen that, the more one wants to be conservative, the more interest their is in reducing uncertainty before making the decision. Evidently, to shield oneself against more scenarios (higher risk coverage), planning and investment costs are naturally higher, see bottom part of Figure 4

The computation of VoI is also repeated for varying level of uncertainty reduction (with fixed $\alpha=0.95$ ), i.e. associated to more or less effective information collection or knowledge acquisition processes, and displayed on Figure 5 .

As can be expected, the more one manages to 


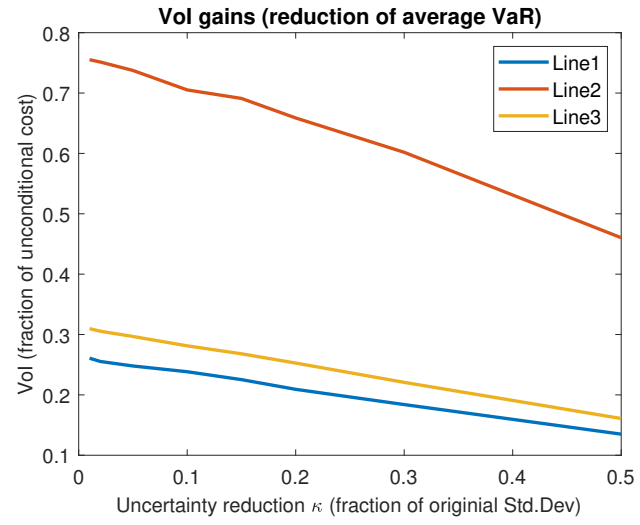

Fig. 5. Evolution of VoI with level of uncertainty reduction

reduce uncertainty before coming to a decision, the more conditional decisions will be preferable, as they are generally more "tailored" to the true situation, to unconditional one, thus driving $\mathrm{VoI}$ upward.

\subsection{General remarks on VoI and robust attitudes}

VoI, as the computed expected difference between the outcomes of unconditional and conditional decisions, is a decision-support metric to guide information collection. Seen from a purely mathematical perspective, it may be viewed as a heuristic in the context of a large scale optimization problem where collecting information before deciding, rather than acting directly, may yield better outcomes. As information collection may itself generate a cost, this large scale problem involves a tradeoff between information collection and action, a so-called exploration versus exploitation tradeoff, see e.g. Russell and Norvig (2016).

Seen from the perspective of statistical decision theory, one may also call it a stopping problem. No matter how the body of knowledge on which any engineering decision may be based is obtained, enriching it through testing, modeling, learning, has to be considered and the final quality of that body of knowledge has to be questioned, up to a certain point, which is hard to determine. VoI is a possible tool for that purpose.

Generally speaking, improved knowledge on the uncertain environment, insofar as its acquisition cost does not exceed its benefit or if such added cost may be traded off for a less risky lottery, is a crucial ingredient for increased robustness, that is, better control on risk or diminished sensitivity to non-controlled parameters.

\section{Conclusion}

In this paper, it has been proposed to use the concept of Value of Information (VoI) to evaluate, based on the comparison of unconditional and conditional outcomes (more precisely Value-atRisk) of Robust or risk-constrained Optimization (RO), possible gains, either in terms of cost or in terms of risk control, that may be achieved if a reduction of uncertainty is performed before a decision is made.

In refining knowledge and deciding conditionally, provided it is possible for the considered application, the decision-maker may increase robustness in the sense of increasing its control on risk. In particular, it has been seen that the more conservative (risk-adverse) one wants to be, the more valuable it is to reduce uncertainty before deciding.

VoI represents a helpful decision-support metric to elaborate a tradeoff between costly knowledge improvement and lowered operation cost or increased control on risk. The question on when to stop questioning and trying to improve the quality of the body of knowledge on which complex decisions may be based, whether it implies more testing, more modeling efforts or more domain specific understanding, is assuredly a very difficult mathematical one. The objective of the proposed framework is to help addressing it. Then and necessarily, any particular answer, involving the aforementioned tradeoffs, namely exploration versus exploitation, risk versus expected reward, will be dependent on the considered application.

In practice and in continuation of the conceptual discussion proposed in this paper, computation of $\mathrm{VoI}$ in the context of RO, as an imbrication of stochastic optimization and numerical integration schemes (double-loop problem), is a serious computational challenge. Additionally the resolution of the RO problem itself is a serious difficulty for many applications, e.g. for complex systems with decision vectors of large dimension, or for time-varying problems with sequential decisionmaking involved. Such difficulties constitute perspectives of the present work.

\section{References}

Aven, T. (2012). Foundations of risk analysis. John Wiley \& Sons.

Bedford, T., R. Cooke, et al. (2001). Probabilistic risk analysis: foundations and methods. Cambridge University Press.

Berger, J. O. (2013). Statistical decision theory and bayesian analysis. Springer Science \& Business Media.

Charnes, A. and W. W. Cooper (1959). Chanceconstrained programming. Management science 6(1), 73-79.

Cox Jr, L. A. (2012). Confronting deep uncertainties in risk analysis. Risk Analysis: An International Journal 32(10), 1607-1629.

Fauriat, W. and E. Zio (2018). An importance measure to assess the value of a component 
inspection policy. In 2018 3rd International Conference on System Reliability and Safety (ICSRS), pp. 368-375. IEEE.

Howard, R. A. (1966). Information value theory. IEEE Transactions on systems science and cybernetics 2(1), 22-26.

Kaplan, S. and B. J. Garrick (1981). On the quantitative definition of risk. Risk analysis 1(1), 11-27.

Memarzadeh, M. and M. Pozzi (2016). Value of information in sequential decision making: component inspection, permanent monitoring and system-level scheduling. Reliability Engineering \& System Safety 154, 137-151.

Mulvey, J. M., R. J. Vanderbei, and S. A. Zenios (1995). Robust optimization of large-scale systems. Operations research 43(2), 264-281.

Pozzi, M. and A. Der Kiureghian (2011). Assessing the value of information for long-term structural health monitoring. In Health monitoring of structural and biological systems 2011, Volume 7984, pp. 79842W. International Society for Optics and Photonics.

Raiffa, H. (1961). Applied statistical decision theory. Div. of Research, Graduate School of Business Administration, Harvard Univ.

Russell, S. J. and P. Norvig (2016). Artificial intelligence: a modern approach. Malaysia; Pearson Education Limited,.

Savage, L. J. (1972). The foundations of statistics. Courier Corporation.

Straub, D. (2014). Value of information analysis with structural reliability methods. Structural Safety 49, 75-85.

Zonta, D., B. Glisic, and S. Adriaenssens (2014). Value of information: impact of monitoring on decision-making. Structural Control and Health Monitoring 21(7), 1043-1056. 